\title{
Individualized 4-dimensional computed tomography proton treatment for pancreatic tumors
}

\author{
Matthew L Knecht, Ning Wang, April Vassantachart, Rachel Mifflin, Jerry D Slater, Gary Y Yang \\ James M. Slater, MD Proton Treatment and Research Center, Loma Linda University Medical Center, Loma Linda, CA, USA \\ Contributions: (I) Conception and design: ML Knecht, GY Yang, N Wang, JD Slater, A Vassantachart; (II) Administrative support: GY Yang, JD Slater; \\ (III) Provision of study material or patients: GY Yang, JD Slater; (IV) Collection and assembly of data: ML Knecht, GY Yang, N Wang, A Vassantachart, \\ R Mifflin; (V) Data analysis and interpretation: ML Knecht, GY Yang, N Wang, A Vassantachart, R Mifflin; (VI) Manuscript writing: All authors; \\ (VII) Final approval of manuscript: All authors. \\ Correspondence to: Matthew L Knecht. James M. Slater, MD Proton Treatment and Research Center, Loma Linda University Medical Center, 11234 \\ Anderson St, Loma Linda, CA 92354, USA. Email: mknecht@llu.edu.
}

\begin{abstract}
Background: The goal of this study is to determine whether a phase or reconstruction of a 10-phase 4 dimensional computed tomography (4D CT) scan can be used as the primary planning scan for proton treatment of the pancreas, thus eliminating the need for second a slow CT or free breathing CT.

Methods: Ten patients with pancreatic adenocarcinoma were simulated with 4D CT and a proton treatment plan generated based upon one of three primary planning scans, the T0 phase, T50 phase or average reconstruction. These plans were then exported to each of the remaining phases of the 4D CT and the dose to $95 \%$ of the target (D95) calculated. Plans were deemed adequate if the D95 remained at $99 \%$ of the prescribed dose or greater.

Results: For the ten patients in this study anterior abdominal motion was found to range from $2-27 \mathrm{~mm}$ (mean $7.50 \pm 6.79 \mathrm{~mm}$ ). For 9 of 10 patients the anterior abdominal motion was $\leq 8 \mathrm{~mm}$ and all three primary planning scans provided adequate target coverage, resulting in minimum D95 coverage per plan of T0_plan 99.7\%, T50_plan 99.3\% and AVE_plan 99\%. However no plan provided adequate target coverage on the single patient with the largest anterior abdominal motion, $27 \mathrm{~mm}$, and cranio-caudal motion, $20 \mathrm{~mm}$, with minimum D95 values of T0_plan 96.3\%, T50_plan 68\%, and AVE_plan 68\%.

Conclusions: The primary plans tested based up on the T0, T50 and average reconstructions provided adequate D95 coverage throughout the respiratory cycle as long as the anterior abdominal motion was $\leq 8 \mathrm{~mm}$ and can be considered for use as the primary proton planning scan.
\end{abstract}

Keywords: Proton; stereotactic body radiotherapy; 4 dimensional computed tomography (4D CT)

Submitted Oct 14, 2016. Accepted for publication Dec 16, 2016.

doi: 10.21037 /jgo.2016.12.10

View this article at: http://dx.doi.org/10.21037/jgo.2016.12.10

\section{Introduction}

The utilization of proton therapy has increased sharply with an increasing number of proton centers opening within the United States. The challenges of accurately delivering particle therapy increase over photon based techniques due to the Bragg peak and sharp energy fall off. Thus as intrafractional motion occurs, the water-equivalent path length (WPL) can vary as organs of different density pass through the particle's path, resulting in under coverage of the planning tumor volume (PTV) and higher doses to organs at risk (1-3).

The ability to deliver a planned radiation dose, robustness, of a treatment plan depends on factors which cannot be entirely accounted for by a single free breathing planning CT (4). These factors are eloquently described in the Paganetti paper and include factors independent of 
the dose calculation including compensator design, patient setup, and beam reproducibility, as well as uncertainty from dose calculation (5). Intrafractional motion is predominately due to respiratory motion in the upper abdomen, and causes difficulty creating a robust plan. Active breathing control, other spirometric devices, and inducing apnea under general anesthesia have been tried to eliminate motion (4), however many patients cannot tolerate these interventions (2). Thus 4 dimensional computed tomography (4D CT) scans were developed which allowed target motion to be quantified and margins increased to account for motion.

While the 4D CT has been used to evaluate the motion of the tumor, typically a second CT scan is performed and used as the primary planning scan. At our institution proton treatment planning was developed before the era of 4D CT scanning, thus a slow CT protocol was developed which utilized a step and shoot technique, advancing after completing one complete revolution. This provides both a planning CT and an estimation of the motion. On other protocols a second free breathing scan is done as seen on the Radiation Therapy Oncology Group (RTOG) 1112. This adds to patient dose, cost, and resource utilization that could otherwise be spared if a phase or reconstruction of the 4D CT scan could be used as the planning CT scan.

Several proton groups have since asked the question of whether a portion of the 4D CT scan can be used as the primary scan for dose calculation. Kang et al. evaluated ten patients with mobile lung tumors and found that a modified average CT provided an effective strategy for lung treatment planning (6). Similarly Wang et al. reviewed 12 mobile lung tumors developing a planning scan based upon a 3 phase maximum intensity projection (7). In the distal esophagus, Pan et al. reviewed five patients with the diaphragm in the beam path. They evaluated plans developed from the maximum inspiration phase, maximum expiration phase, and the average reconstruction and found that the maximum inspiration phase along with a smearing margin lead to adequate target coverage (2).

However the appropriate phase or reconstruction of a 4D CT scan for pancreatic tumors has not to our knowledge been evaluated. Therefore our group asked if there is a phase or reconstruction of the 4D CT which can replace the second CT scan for pancreatic proton treatment planning.

\section{Methods}

Ten patients with pancreatic cancer were treated with stereotactic body proton radiation therapy at our institution from January to December 2015. For the purposes of this study, each patient was simulated using a full body immobilization device while 4D CT images were acquired. The device consisted of a carbon fiber pod filled with a selfexpanding foam that molds to the external contour of the patient. This reproducible immobilization system forms around the posterior aspect of the patient and covers a $180^{\circ}$ area, which creates a reproducible external contour (8). A multi-slice scanner acquired the 4D CT images while the patient was immobilized. This involved an external fiducial to monitor the patient's respiratory cycle during image acquisition where ten data sets were developed (referred to as T0, T10, T20,..., T90) that were equally divided between T0 (maximum inhalation) and T50 (maximum exhalation). An average CT was also created by computing the average Hounsfield unit in each voxel merged over all ten scans.

\section{Target delineation}

Gross tumor volume (GTV) was contoured on each of the ten 4D CT phases with contrast, utilizing the treatment planning system Odyssey 5.1 (Optivus, San Bernardino, CA, USA). Clinical target volume (CTV) was equivalent to the GTV without expansion, as delineated by a radiation oncologist, without deformable image registration. The internal target volume (ITV) was generated by forming a union of the GTV contours from all ten individual phases of the 4D CT. A set up margin of $5 \mathrm{~mm}$ was added for set up uncertainty that was achievable with pod immobilization and daily orthogonal $\mathrm{kV}$ imaging. Normal structures including the stomach, spinal cord, kidneys, liver, duodenum, small bowel, and colon were manually contoured on all ten phases as well as on the average data set images from the 4D CT per RTOG Upper Abdominal Normal Organ Contouring Consensus guidelines (9).

\section{Proton treatment plans}

A dose of 30 Gy in 5 fractions was prescribed to D95, such that $100 \%$ of the prescribed dose was given to $95 \%$ of the volume of the ITV plus setup margin, with beam specific PTV margin added by the planning software. The target was covered with 2-3 coplanar proton beams with priority given to beams between the right lateral and posterior positions, which enter through pod. This utilized the 
Table 1 Baseline patient characteristics

\begin{tabular}{lccccc}
\hline Patient & L-R motion $(\mathrm{mm})$ & A-P motion $(\mathrm{mm})$ & CC motion $(\mathrm{mm})$ & Ant abdominal motion $(\mathrm{mm})$ & ITV $(\mathrm{mm})$ \\
\hline A & 1.9 & 3.0 & 10 & 8 & $41 \times 39$ \\
B & 7.8 & 3.9 & 7.5 & 7 & $52 \times 44$ \\
C & 9.8 & 5.9 & 15 & 7 & $52 \times 42$ \\
D & 4.9 & 4.9 & 5 & 6 & $35 \times 27$ \\
E & 2.9 & 3.9 & 7.5 & 3 & $24 \times 23$ \\
F & 4.0 & 6.9 & 12.5 & 3 & $54 \times 53$ \\
G & 6.9 & 2.8 & 7.5 & 27 & $45 \times 41$ \\
H & 3.0 & 5.9 & 7.5 & 2 & $42 \times 37$ \\
I & 3.0 & 3.9 & 10 & 5 & $43 \times 41$ \\
J & 4.0 & 3.0 & 7 & $36 \times 30$ \\
\hline
\end{tabular}

L-R, left-right; A-P, anterior-posterior; CC, cranial caudal; ant, anterior; ITV, internal target volume.

consistent external WPL due to the immobilization device, unless patient normal organ constraints required a beam outside of this arc. Beam characteristics (e.g., aperture, range, bolus, etc.) were managed by methods previously described by Moyers et al. (10) and density corrections were applied to correct for contrast and air inhomogeneities. A plan was then developed for each of three primary scans; the T0 phase (maximum inspiration), the T50 phase (maximum expiration) and the average CT (hereafter referred to as T0_plan, T50_plan, and AVE_plan). The beam angles, beam characteristics, and dose were translated unaltered to each of the ten phases of the 4D CT.

\section{Plan analysis}

To evaluate each plan (T0_plan, T50_plan and AVE_plan) the beams and beam characteristics were fixed and then imported into each of the 10 phases of the 4D CT. In each phase, the GTV was outlined and a setup margin added. This volume, representing the tumor dose in that phase, was evaluated for coverage. A plan was defined as adequate if the D95 coverage was no lower than $99 \%$ of the prescribed dose on any of the 10 phases of the 4D CT (2). Cranialcaudal motion was determined on 4D CT by measuring the displacement of the center of mass of the tumor on each phase and abdominal motion was determined on the average scan as the region of gradient hypodensity directly over the center of mass of the tumor.

\section{Results}

Baseline patient characteristics are shown in Table 1. The range, mean, and standard deviation of tumor motion were as follows: left-right dimension, range 1.9-9.8 $\mathrm{mm}$, mean $4.8 \mathrm{~mm}$, standard deviation $2.5 \mathrm{~mm}$, anterior-posterior, range $3-8.8 \mathrm{~mm}$, mean $5.0 \mathrm{~mm}$, standard deviation $1.9 \mathrm{~mm}$, and in the superior-inferior dimension range of $5-20 \mathrm{~mm}$, mean $10 \mathrm{~mm}$, standard deviation of $4.5 \mathrm{~mm}$. Tumor sizes ranged from 21 to $52 \mathrm{~mm}$ in the largest axial diameter.

Treatment plans (T0_plan, T50_plan and AVE_plan) were successfully generated for all patients. We then tested these three plans on all ten phases of the 4D CT and results showed that adequate target coverage, D 95 covered by $99 \%$ of the prescribed dose or greater, was achieved for 9 of the 10 patients as seen in Table 2. For these nine patients the minimum coverage per plan was T0_plan 99.7\%, T50_ plan $99.3 \%$ and AVE_plan $99 \%$. Patient E was chosen as a representative patient with abdominal motion of $3 \mathrm{~mm}$ and cranio-caudal motion of $7.5 \mathrm{~mm}$, and the D95 coverage per phase and plan is shown in Table 3. Individual phases and patient coverage is shown in Figures 1-3.

Correlation between cranio-caudal and anterior abdominal motion was minimal (Spearman's rho $r$ value $0.513,2$-tailed significance 0.13 ). However, for 9 of the 10 patients, the tumor motion ranged from $5-12.5 \mathrm{~mm}$ (mean $9.17 \pm 2.89 \mathrm{~mm}$ ) and abdominal motion ranged from 3-8 mm (mean $5.33 \pm 2.05 \mathrm{~mm}$ ). Patient $\mathrm{G}$ had the largest motion in both dimensions, at $20 \mathrm{~mm}$ in cranio-caudal 
Table 2 Range of the minimum D95 coverage per plan for each patient

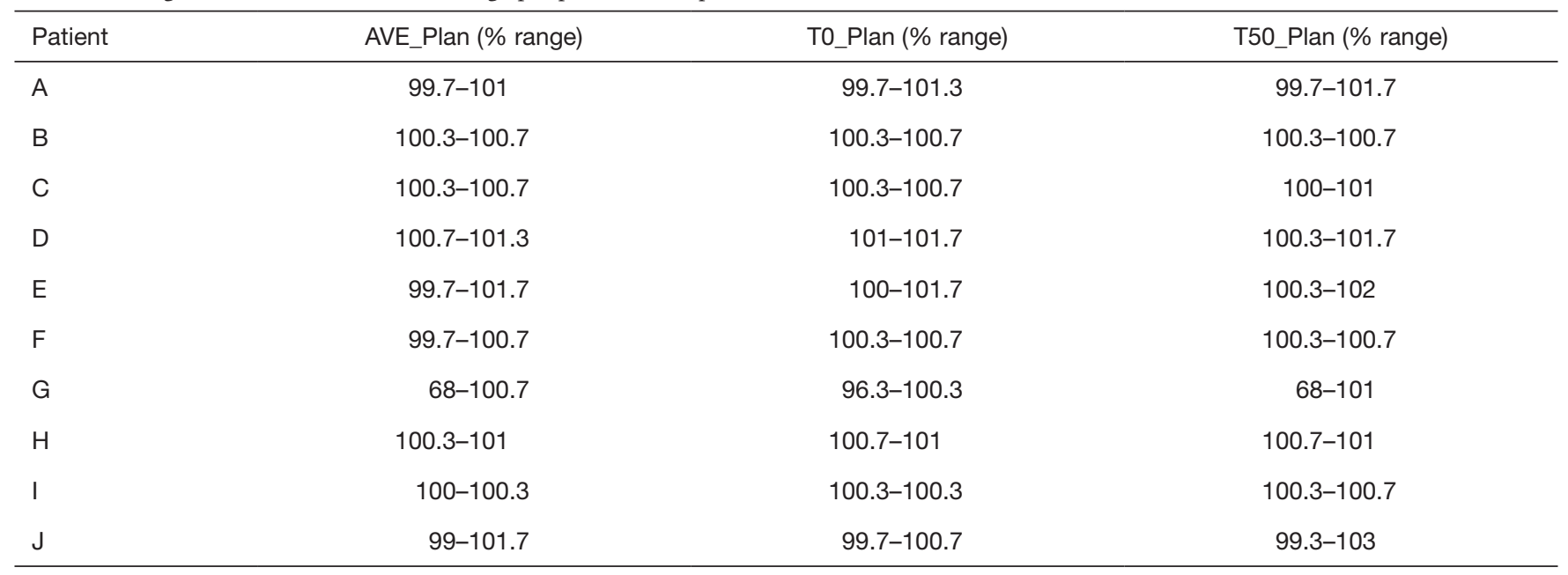

AVE, average.

Table 3 Representative of the nine adequately covered patients, Patient E's percent D95 coverage is shown

\begin{tabular}{lrcc}
\hline \multirow{2}{*}{ Phase } & \multicolumn{3}{c}{ Plan $(\%)$} \\
\cline { 2 - 4 } & AVE & T0 & T50 \\
\hline T0 & 100.7 & 101.0 & 101.0 \\
T10 & 101.3 & 101.7 & 101.7 \\
T20 & 101.7 & 101.7 & 102.0 \\
T30 & 101.3 & 101.7 & 101.7 \\
T40 & 101.3 & 101.7 & 101.7 \\
T50 & 99.7 & 100.0 & 101.0 \\
T60 & 101.3 & 101.3 & 101.3 \\
T70 & 101.0 & 101.3 & 101.3 \\
T80 & 100.7 & 101.0 & 101.3 \\
T90 & 99.7 & 100.0 & 100.3 \\
\hline
\end{tabular}

AVE, average.

tumor motion and $27 \mathrm{~mm}$ in abdominal motion. Patient G's large abdominal and cranio-caudal motion was due to difficultly in controlling celiac axis pain.

Results for the single patient (patient G), showed that for the T50_plan (max expiration) and the AVE_plan, target coverage was less than $99 \%$ the prescription dose in the phases centered on maximum inspiration [T0 ( $\max$ inspiration), T10, T80, T90]. The T0_plan provided coverage below $99 \%$ of the prescribed dose on the T20,
T30, T40, T50, T60 and T70 phases as seen in Table 4 and Figure 4.

\section{Discussion}

To our knowledge this represents the first data for utilizing 4D CT as the primary proton treatment planning scan. Our results show that for patients with less than $8 \mathrm{~mm}$ of anterior abdominal motion, the T0, T50, or average phases of the 4D CT produced acceptable target coverage over the 10 phases of the $4 \mathrm{D} \mathrm{CT}(2,11)$. This data provides initial evidence that using planning techniques for bolus design, smearing parameters, blocks, and modulation wheel selection as described by Moyers et al. (10) are sufficient to accommodate for WPL changes due to respiration for tumors of the pancreas.

To determine the applicability of this data, reviews of other studies which have evaluated pancreatic motion show differing results. Tai $e t$ al. analyzed pancreatic motion utilizing 4D CT for 15 patients. They initially evaluated 12 patients selected at random who had a cranio-caudal tumor motion of $5.8 \pm 3.1 \mathrm{~mm}$, while a second selected group of patients were chosen for their large respiratory motion and found to have a cranio-caudal motion of $11 \pm 1.4 \mathrm{~mm}$ (12). Another analysis by Hallman et al. (13) showed that for pancreatic tumors measured with 4D CT, the center of mass of the CTV had a cranio-caudal motion of $5 \pm 1 \mathrm{~mm}$. Our study included patients with what was primarily considered a large respiratory motion, with cranio-caudal motion found 


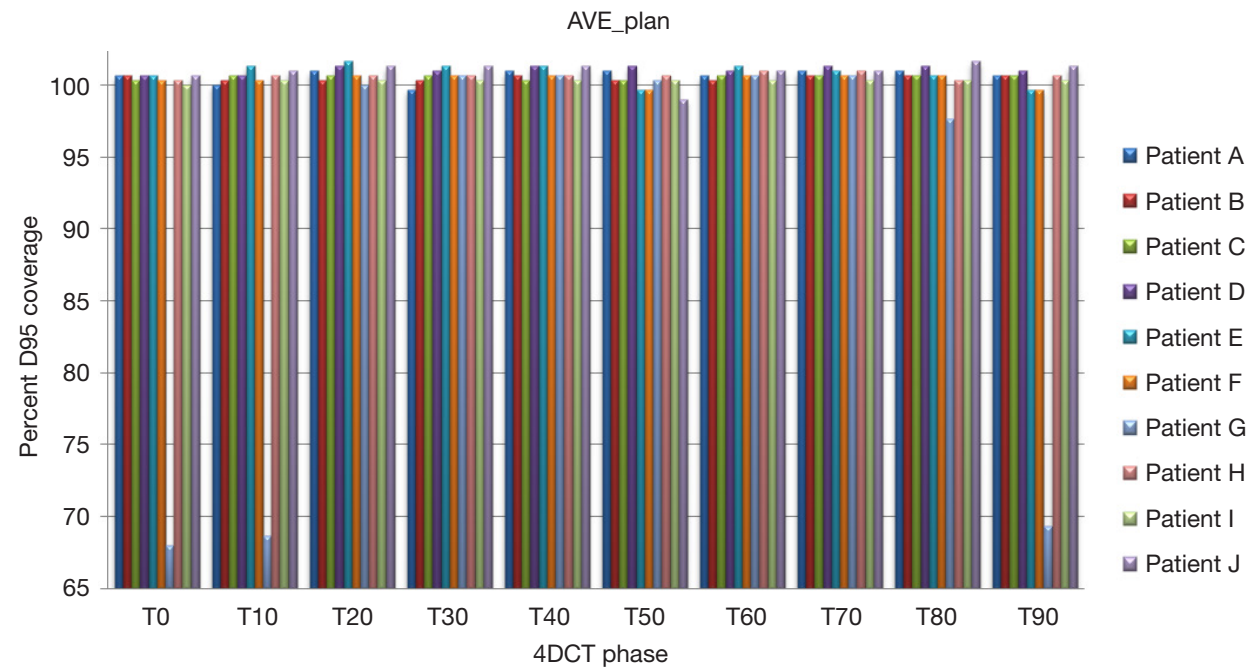

Figure $1 \mathrm{D} 95 \%$ for each patient per respiratory phase as seen with the average plan. AVE, average; 4DCT, 4 dimensional computed tomography.

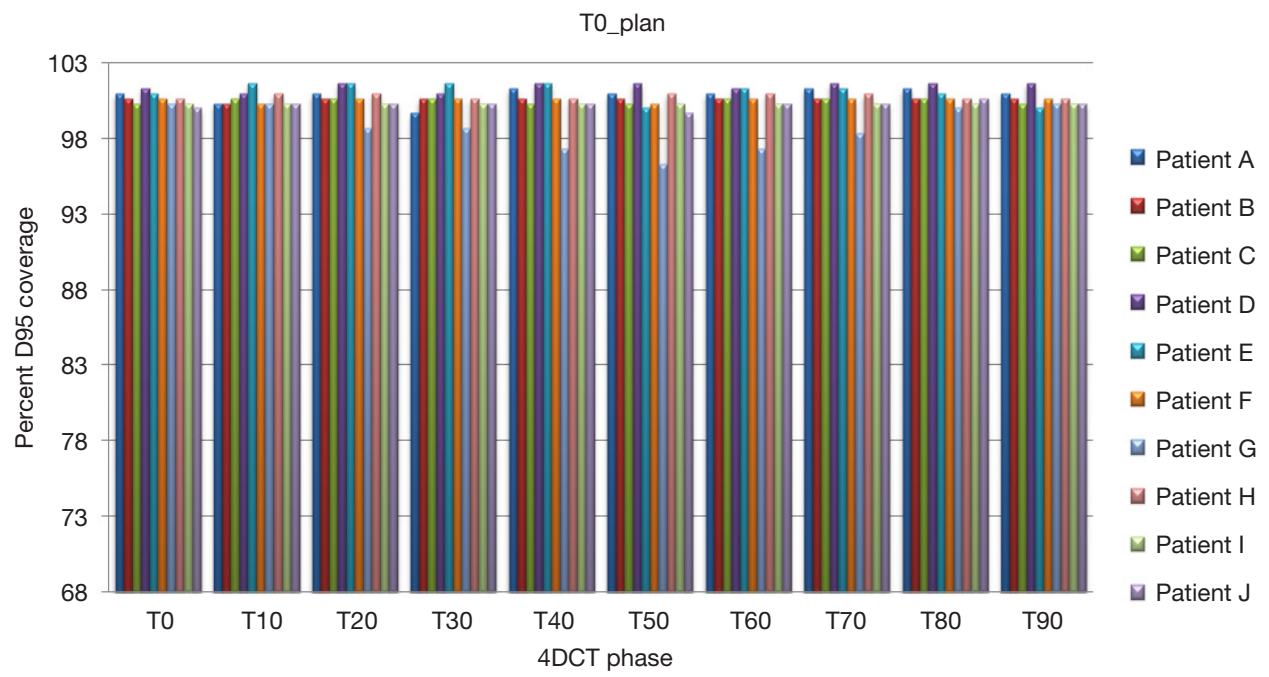

Figure 2 D95\% for each patient per respiratory phase as seen with the T0 plan. 4DCT, 4 dimensional computed tomography.

to be $10.3 \pm 4.3 \mathrm{~mm}$, and thus covers the range of motion for most pancreatic tumors.

However for patients with large abdominal wall motion, such as patient $\mathrm{G}$ in our study, caution must be used. Patient $\mathrm{G}$ demonstrated $27 \mathrm{~mm}$ of abdominal motion and $20 \mathrm{~mm}$ of cranio-caudal tumor motion. The plan did not meet the specified $99 \%$ dose coverage due to the large abdominal motion and the use of an anterior beam. In our study the planners were allowed to use anterior beam angles per our institution practice to spare normal organs, and anterior beams were used in patients B, C, D, F, and G. In the case of patient $\mathrm{G}$, the degree of anterior abdominal wall motion caused all three plans to fail. For the T50_plan and AVE_ plan the plan provided inadequate distal coverage of the PTV at maximum inspiration due to abdominal distension and increased WPL to the target. The T0_plan showed improved target coverage throughout the ten phases (maximum under dose: T50_plan 32\%, AVE_plan 32\%, T0_plan 3.7\%), however, during maximum exhalation the proximal target coverage was inadequately covered due to overshoot of the proton beam, as the WPL to the target was reduced. The other nine cases had abdominal wall motion 


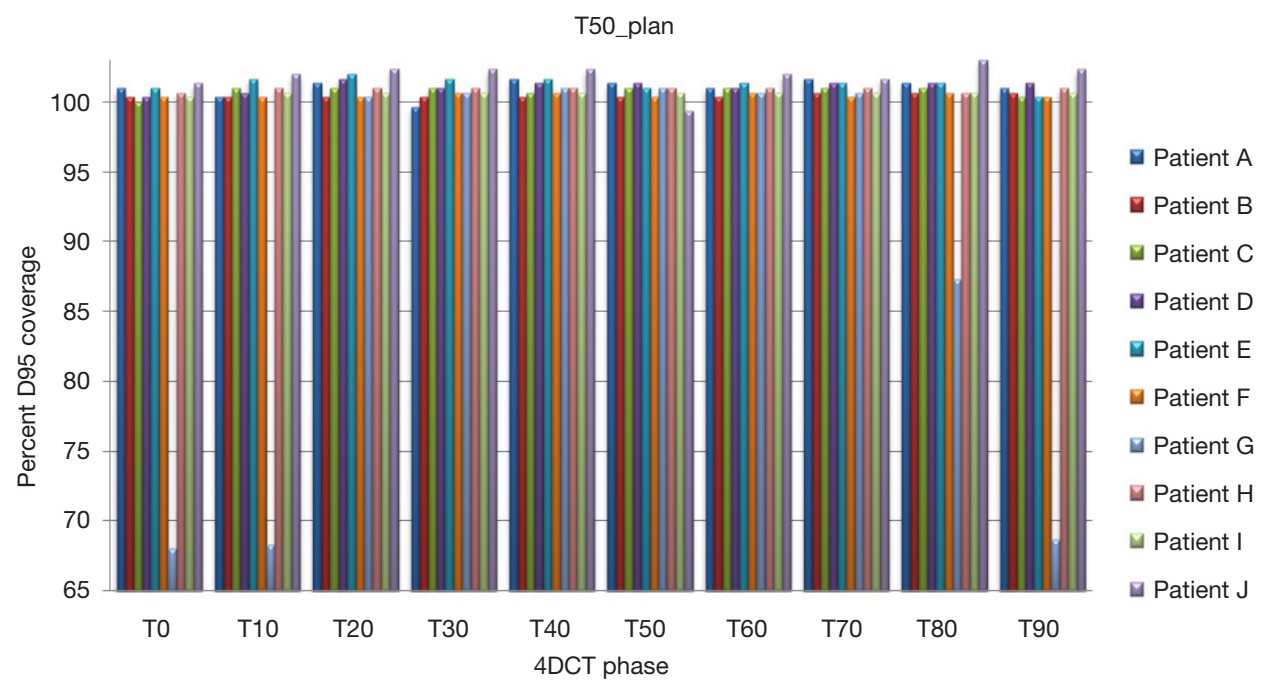

Figure 3 D95\% for each patient per respiratory phase as seen with the T50 plan. 4DCT, 4 dimensional computed tomography.

Table 4 Patient G's percent D95 coverage

\begin{tabular}{lccc}
\hline \multirow{2}{*}{ Phase } & \multicolumn{3}{c}{ Plan $(\%)$} \\
\cline { 2 - 4 } & AVE & T0 & T50 \\
\hline T0 & 68.0 & 100.3 & 68.0 \\
T10 & 68.7 & 100.3 & 68.3 \\
T20 & 100.0 & 98.7 & 100.3 \\
T30 & 100.7 & 98.7 & 100.7 \\
T40 & 100.7 & 97.3 & 101.0 \\
T50 & 100.3 & 96.3 & 101.0 \\
T60 & 100.7 & 97.3 & 100.7 \\
T70 & 100.7 & 98.3 & 100.7 \\
T80 & 97.7 & 100.0 & 87.3 \\
T90 & 69.3 & 100.3 & 68.7 \\
\hline
\end{tabular}

AVE, average.

of $<8 \mathrm{~mm}$ and standard techniques were able to account for the variation involved.

As the use of an anterior beam was thought to lead to the failure of all three plans, patient $G$ was replanned without an anterior beam. This plan showed minimum percent D95 target coverage per plan of T50_plan $100.3 \%$, T0_plan $100.3 \%$ and AVE_plan $100 \%$. Thus to address patients with anterior abdominal motion larger than $8 \mathrm{~mm}$, avoidance of anterior beams could be a reasonable approach. If anterior beams are required to spare normal organs (kidney, liver), then respiratory gaiting can be used to treat the lesion only during the phases where adequate target coverage is achieved. Abdominal compression could also be employed, which has been shown to significantly reduce craniocaudal motion (14), however have not yet been studied for anterior abdominal motion. Finally, if no such methods are available the T0_plan provided the highest degree of coverage (minimum D95 covered by $96.3 \%$ ), and could be considered.

Pan et al. (2) evaluated both which phase of the 4D CT to use for treatment planning as well as which smearing diameter to use to create the most robust proton plan for distal esophageal tumors. The distal esophagus was chosen as a region which had significant motion, $20-48 \mathrm{~mm}$, and WPL changes in the five patients studied. Similar to this analysis, Pan et al. exported the plan to the ten phases of the 4D CT and found that the T0_plan provided a 99\% prescription dose coverage of the ITV throughout all phases of the 4D CT, while the T50 plan and AVE_plan were unable to meet this criteria. In both our study and the Pan analysis 4D CT was able to provide an adequate proton treatment plan, and our study delineated that each phase studied provided an adequate plan given the anterior abdominal motion was less than or equal to $8 \mathrm{~mm}$.

The limitations of this study include the small patient number, lack of a validation cohort, and need to obtain a 4D CT before motion can be analyzed. The patient population included nine patients who were adequately planned with motion between $2-8 \mathrm{~mm}$ and only one patient who had inadequate coverage at with $27 \mathrm{~mm}$ of motion. Thus the 

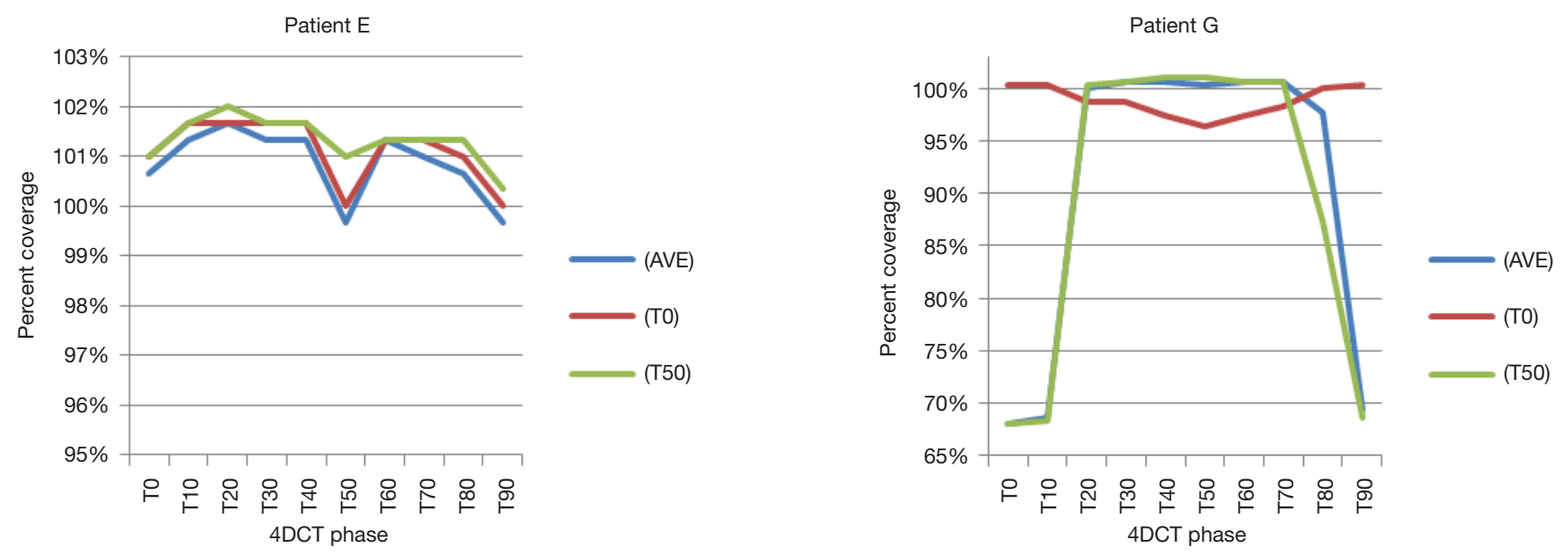

Figure 4 Patients $\mathrm{E}$ and $\mathrm{G}$ target coverage by phase showing patient $\mathrm{G}$ inadequate coverage by phase, while patient $\mathrm{E}$ maintains adequate coverage throughout. AVE, average; 4DCT, 4 dimensional computed tomography.

need to dampen abdominal motion between 9-27 mm remains unclear. Also having a second, independent cohort of patients with less than or equal to $8 \mathrm{~mm}$ of motion which are able to be planned with adequate D95 target coverage would help to showing the validity of the findings of this study. In keeping with our goal to reduce the number of scans done, as motion is analyzed on the 4D scan, a second scan is required if the motion is significant and abdominal compression is used.

\section{Conclusions}

Each of the phases studied, T0, T50 and Average, produced a plan which provided acceptable results and can be used for proton treatment planning in the treatment of pancreatic malignancy as long as abdominal motion is less than or equal to $8 \mathrm{~mm}$. Implementing the strategy of utilizing a single phase of the 4D CT will allow a reduction of dose to the patient, CT simulator time, and cost for pancreatic treatments, while still maintaining adequate target coverage.

\section{Acknowledgements}

The authors would like to acknowledge Dr. Baldev Patyal for his critique of the study design and guidance.

\section{Footnote}

Conflicts of Interest: The authors have no conflicts of interest to declare.
Ethical Statement: The study was approved by institutional ethics board and approved per IRB\#53191 and written informed consent was obtained from all patients.

\section{References}

1. Mori S, Wolfgang J, Lu HM, et al. Quantitative assessment of range fluctuations in charged particle lung irradiation. Int J Radiat Oncol Biol Phys 2008;70:253-61.

2. Pan X, Zhang X, Li Y, et al. Impact of using different fourdimensional computed tomography data sets to design proton treatment plans for distal esophageal cancer. Int J Radiat Oncol Biol Phys 2009;73:601-9.

3. Engelsman M, Rietzel E, Kooy HM. Four-dimensional proton treatment planning for lung tumors. Int J Radiat Oncol Biol Phys 2006;64:1589-95.

4. Engelsman M, Schwarz M, Dong L. Physics controversies in proton therapy. Semin Radiat Oncol 2013;23:88-96.

5. Paganetti H. Range uncertainties in proton therapy and the role of Monte Carlo simulations. Phys Med Biol 2012;57:R99-117.

6. Kang Y, Zhang X, Chang JY, et al. 4D Proton treatment planning strategy for mobile lung tumors. Int $\mathrm{J}$ Radiat Oncol Biol Phys 2007;67:906-14.

7. Wang N, Patyal B, Ghebremedhin A, et al. Evaluation and comparison of New 4DCT based strategies for proton treatment planning for lung tumors. Radiat Oncol 2013;8:73.

8. Wroe AJ, Bush DA, Schulte RW, et al. Clinical immobilization techniques for proton therapy. Technol 
Cancer Res Treat 2015;14:71-9.

9. Jabbour SK, Hashem SA, Bosch W, et al. Upper abdominal normal organ contouring guidelines and atlas: a Radiation Therapy Oncology Group consensus. Pract Radiat Oncol 2014;4:82-9.

10. Moyers MF, Miller DW, Bush DA, et al. Methodologies and tools for proton beam design for lung tumors. Int J Radiat Oncol Biol Phys 2001;49:1429-38.

11. Velec M, Moseley JL, Dawson LA, et al. Dose escalated liver stereotactic body radiation therapy at the mean respiratory position. Int J Radiat Oncol Biol Phys 2014;89:1121-8.

Cite this article as: Knecht ML, Wang N, Vassantachart A, Mifflin R, Slater JD, Yang GY. Individualized 4-dimensional computed tomography proton treatment for pancreatic tumors. J Gastrointest Oncol 2017;8(4):675-682. doi: 10.21037/ jgo.2016.12.10
12. Tai A, Liang Z, Erickson B, et al. Management of respiration-induced motion with 4-dimensional computed tomography (4DCT) for pancreas irradiation. Int J Radiat Oncol Biol Phys 2013;86:908-13.

13. Hallman JL, Mori S, Sharp GC, et al. A four-dimensional computed tomography analysis of multiorgan abdominal motion. Int J Radiat Oncol Biol Phys 2012;83:435-41.

14. Lovelock DM, Zatcky J, Goodman K, et al. The effectiveness of a pneumatic compression belt in reducing respiratory motion of abdominal tumors in patients undergoing stereotactic body radiotherapy. Technol Cancer Res Treat 2014;13:259-67. 